\title{
Secondary syphilis: Hepatitis, rashes and prozone phenomenon
}

\author{
Abdurrahman Kaya ${ }^{1 *}$, Sibel Yıldız Kaya ${ }^{2}$, Ahmet Furkan Kurt ${ }^{3}$, Meryem Şahin $^{3}$ and Ali Can ${ }^{4}$ \\ ${ }^{1}$ Department of Infectious Diseases, İstanbul Training and Research Hospital, Turkey \\ ${ }^{2}$ Department of Infectious Disease, Sungurlu State Hospital, Turkey \\ ${ }^{3}$ Department of Infectious Disease, Medical School of Cerrahpasa, Istanbul University, Turkey \\ ${ }^{4}$ Department of Internal Medicine, Division of Immunology and Allergic disease, Istanbul School of Medicine, Istanbul University, Turkey
}

\begin{abstract}
Syphilis is a sexually transmitted disease. Sometimes, it is challenging to diagnose due to false negative serologic results. Therefore, the proper use and interpretation of diagnostic testing are important for optimal patient management. Prozone phenomenon is a very rare and false negative reaction and occurs due to disproportionately increased antibodies in a specimen. Immune system is responsible for excess antibodies. It is frequently seen in HIV, neurosyphilis, early syphilis (mainly secondary syphilis). This reaction causes delays in the diagnosis and treatment of the disease. Thus, in cases where VDRL is non-reactive, it is important for clinicians to consider prozone reaction in patients with clinical signs suggesting syphilis. Here, we present a case of secondary syphilis diagnosed late due to prozone phenomenon.
\end{abstract}

\section{Introduction}

Syphilis is a sexually transmitted disease, early diagnosis and treatment are essential for control and prevention of the disease. Serologic antibody studies are the most commonly used methods for its diagnosis. These include the use of both non-treponemal assays for screening and treponemal assays for confirmation. Since false positive and negative results can be seen by serology, the proper use and interpretation of diagnostic testing are important for optimal patient management. Prozone phenomenon (PP) is a very rare and false negative reaction. It is frequently seen in HIV, neurosyphilis, early syphilis (mainly secondary syphilis) and causes delays in the diagnosis and treatment of the disease [1,2]. Here, we present a case of secondary syphilis diagnosed late due to PP.

\section{Case report}

A 79-year-old man was admitted to outpatient dermatology clinic with 4 weeks of fatigue, weight loss (15 kilograms) and rashes. They were faint, erythematous, ovaloid macular eruptions involving bilateral the feet and the hands. He denied fever and adenopathy. Outside laboratory evaluation found a white blood cell count of $7500 / \mu \mathrm{l}$, C-reactive protein of $81 \mathrm{mg} / \mathrm{l}$, erythrocyte sedimentation rate of 111 $\mathrm{mm} /$ hour, Alanine aminotransferase (ALT): $523 \mathrm{IU} / \mathrm{L}$ (normal 0-50 IU/L), Aspartate aminotransferase (AST): $561 \mathrm{IU} / \mathrm{L}$ (normal 0-50 IU/L), elevated gamma glutamyl transferase (GGT): 520 IU/L (normal 0-38 IU/L), elevated alkaline phosphatase (ALP): $739 \mathrm{IU} / \mathrm{L}$ (normal 30-120 IU/L), Total bilirubin: $0.32 \mathrm{mg} / \mathrm{dl}$ (normal $0.3-1.2 \mathrm{mg} / \mathrm{dl}$ ) and anemia. Serology for Venereal disease research laboratory (VDRL), Hepatitis B Virus, HIV and Hepatitis C Virus, Chlamydia trachomatis and Toxoplasmosis remained negative. On the 30th days of symptoms, he referred to our clinic because of abnormal liver enzyme levels. On examination, the lesions were non-itchy, scaly rashes over the palms (Figure 1) and the soles (Figure 2). In his medical history, the patient visited to Russia 4 years ago and had sexual intercourse with many different women. Serologies of HIV, VDRL were repeated but remained negative. Ultrasound showed mild hepatomegaly. On follow-up, the rashes were not resolved, his fatigue continued and the liver function tests were fluctuated. Due to strong suspicion of the syphilitic rashes including soles and palms, we communicated to the laboratory and later VDRL was tested after diluting the sample. VDRL positivity at 1/256 titers were detected. Afterwards, Treponema Pallidum Hemagglutination Antigen (TPHA) was found to be positive at 1/1280 titers. As his wife died, diagnostic testing was not performed. As a result, he was considered secondary syphilis presenting with acute hepatitis, later Penicillin G benzathine (2.4 million units intramuscularly) was administered. His symptoms and signs regressed, and the general condition improved. The normalization of abnormal tests took a month (Table 1). The patient was doing well and no recurrence at the last follow-up visit.

\section{Discussion}

Syphilis is an important infectious disease which presents three clinical stages including primary, secondary and tertiary. If untreated, the bacteria are hematogenously spread all over body in secondary stage. Secondary syphilis develops within weeks to a few months after exposure to the bacterium [3]. During bacterial dissemination, skin, mucous membranes and a wide range of organs including liver can be involved. Adenopathy, fever and weight loss can be developed. However, acute hepatitis is extremely uncommon presentation of the disease and $3 \%$ cases of secondary syphilis are estimated to develop hepatitis [4].

*Correspondence to: Abdurrahman Kaya Department of Infectious Disease, İstanbul Training and Research Hospital, Turkey, E-mail: dr.abdkaya@hotmail.com Key words: syphilis, prozone phenomenon, hepatitis

Received: February 16, 2021; Accepted: February 23, 2021; Published: February 26, 2021 


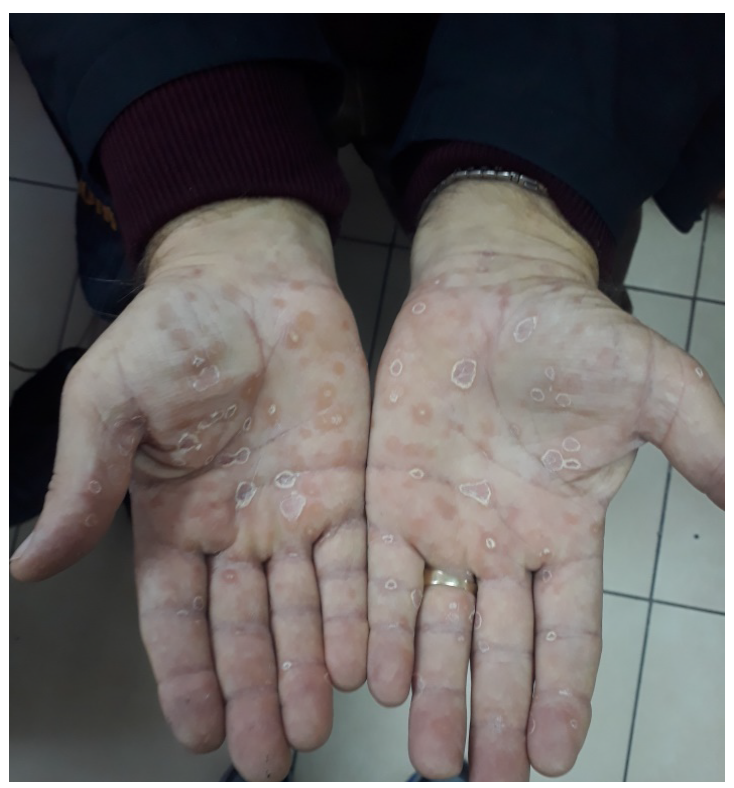

Figure 1. Faint, erythematous, ovaloid scaly rashes over the palms

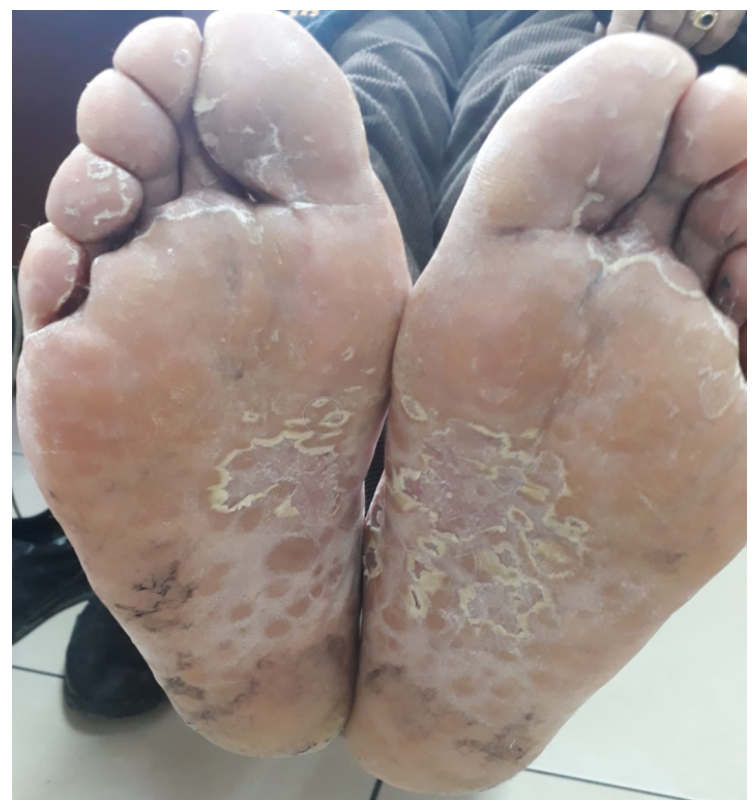

Figure 2. Faint, erythematous, ovaloid scaly rashes over the soles

Table 1. The fluctuation of hepatic enzymes level

\begin{tabular}{|c|c|c|c|c|c|c|c|}
\hline \multicolumn{4}{|c|}{ Pre-treatment } & \multicolumn{4}{c|}{ Post-treatment } \\
\hline ALT & 523 & 350 & 427 & 310 & 159 & 86 & 30 \\
\hline AST & 561 & 533 & 535 & 259 & 240 & 128 & 25 \\
\hline ALP & 739 & 690 & 887 & 550 & 290 & 215 & 144 \\
\hline GGT & 520 & 410 & 442 & 422 & 297 & 117 & 61 \\
\hline
\end{tabular}

Syphilitic hepatitis ( $\mathrm{SH}$ ) has four diagnostic criteria including elevation of liver enzyme levels, positive serology for secondary syphilis, and recovery of hepatic injury after treatment, the exclusion of other cause of liver damage [5]. The case met all of the criteria. Contrary to predominance of elevated AST and ALT, it presents with disproportionately increased alkaline phosphatase and slightly high transaminase [6]. SH generally is mild clinical condition and well responds to appropriate antimicrobial therapy. If the patient had not been diagnosed, a skin or liver biopsy would have been required. From a public health perspective, the consequences of delayed diagnoses cannot be underestimated. Therefore, considering PP is important.

Our patient presented with rashes, acute hepatitis and abnormal blood tests. As secondary syphilis is characterized by a wide range of symptoms/signs and can mimic other infections and immunemediated processes, its diagnosis is often overlooked due to its myriad presentations and the lack of experience. In this patient, VDRL was performed 2 times but remained negative and the patient was not diagnosed with syphilis and not given the standard treatment before. VDRL is a non-treponemal serologic testing which is affected a variety of causes. PP is a rare, false negative reaction due to disproportionately increased antibodies in a specimen. It has been reported 0.3 to $2 \%$ of all cases of syphilis $[1,2]$. PP is prevented by diluting the sample. Diluting the serum reduces the concentration of antibody and allows the agglutination reaction. Most laboratories do not routinely test to prevent PP. Therefore, it is important to inform the laboratory when clinical findings strongly suggest syphilis and non-treponemal serological test results are negative.

In this case, the patient lost excess weight and had severe hepatic injury. The acute-phase reactants including sedimentation rate and C-reactive protein were extremely high levels. These findings were unusual for secondary syphilis. We thought the immune response to the disease was highly active, thus producing excess antibodies. Excessive clinical presentation and active inflammation may be due to excessive immune response, resulting in PP.

\section{Conclusion}

As a conclusion, syphilis is communicable infection that can be presented with a variety of clinical manifestations including hepatitis. Therefore, its prompt diagnosis is essential for early treatment. False negative results in serological diagnosis can delay the diagnosis and treatment of the disease. Thus, in cases where VDRL is non-reactive, it is important for clinicians to consider prozone reaction in patients with clinical signs suggesting syphilis. This case will raise the awareness of the physicians on syphilis.

\section{References}

1. Jurado RL, Campbell J, Martin PD (1993) Prozone phenomenon in secondary syphilis: has its time arrived? Arch Intern Med 153: 2496-2498. [Crossref]

2. Uribe CS, Garcia FA (1998) Neurosyphilis and the prozone effect. Rev Neurol 27: 970-972.

3. Clark EG, Danbolt N (1955) The Oslo study of the natural course of untreated syphilis: An epidemiologic investigation based on a re-study of the Boeck-Bruusgaard material; a review and appraisal. J Chronic Dis 2: 311-344. [Crossref]

4. Adachi E, Koibuchi T, Okame M, Sato H, Kikuchi T, et al. (2013) Liver dysfunction in patients with early syphilis: a retrospective study. J Infect Chemother 19: 180-182. [Crossref]

5. Mandache C, Coca C, Caro-Sampara F, Haberstezer F, Coumaros D, et al. (2006) A forgotten etiology of acute hepatitis in immunocompetent patient: syphilitic infection. J Intern Med 259: 214-215.

6. Young MF, Sanowski RA, Manne RA (1992) Syphilitic hepatitis. J Clin Gastroenterol 15: 174-176. [Crossref]

Copyright: (C2021 Kaya A. This is an open-access article distributed under the terms of the Creative Commons Attribution License, which permits unrestricted use, distribution, and reproduction in any medium, provided the original author and source are credited. 